0009

MIR-146A AN IMPORTANT KEY PLAYER IN BONE METABOLISM

${ }^{1} \mathrm{~V}$ Saferding*, 'M Hofmann, ${ }^{1} \mathrm{JS}$ Brunner, ${ }^{1} \mathrm{MF}$ Militaru, ${ }^{1} \mathrm{~A}$ Puchner, ${ }^{1} \mathrm{~S}$ Hayer, ${ }^{2} \mathrm{M}$ Timmen, ${ }^{2} \mathrm{R}$ Stange, ${ }^{1} \mathrm{JS}$ Smolen, ${ }^{1} \mathrm{~S}$ Blüml. ' Medical University of Vienna, Vienna, Austria; ' University Muenster, Muenster, Germany

\subsection{6/annrheumdis-2018-EWRR2018.9}

Introduction Micro RNAs (miRNAs) play a crucial role in the regulation of bone metabolism. MiR-146a, an important antiinflammatory miRNA, was found to negatively impact osteogenesis and bone regeneration in vitro, by controlling the differentiation of mesenchymal stem cells. But to date the role of miR-146a in bone remodelling, its influence on bone stability and development of osteoporosis is not known.

Objectives The objective of this project is the analysis of the role of miR-146a in bone metabolism.

Methods Systemic bone, tibiae and femur, of wt and miR146a deficient animals was assessed histologically and via $\mu \mathrm{CT}$ analysis, over a period of 3 to 18 months of age. Serum cytokine levels were analysed by Elisa. MRNA expression levels in bone were analysed by qPCR. To induce osteoporosis, ovariectomy (OVX) induced bone loss was performed.

Results When we analysed bone volume of long bones histologically as well as with $\mu \mathrm{CT}$ analysis we detected significantly increased trabecular bone mass in miR-146a deficient compared to wt animals, starting at an age of 6 months. However, cortical thickness of systemic bones from miR-146a knock out animals was significantly reduced compared to control mice. Analysis of serum in aged miR-146a deficient animals displayed elevated activity of bone resorbing osteoclasts as amounts of CTX I in miR-146a $\mathrm{a}^{-/-}$mice were significantly increased compared to wt animals. Q-PCR analysis of important osteoclast as well as osteoblast marker genes in bones ex vivo displayed elevated expression of signature molecules of both cell types in aged miR-146a deficient mice, suggesting a regulatory role of miR-146a in both osteoclasts as well as osteoblasts. When we induced osteoporosis using the OVX disease model, histological analysis of long bones showed significant trabecular bone loss in ovariectomized wt mice. In contrast, we detected no trabecular bone loss in ovariectomized miR-146a knock out animals, suggesting that loss of miR146a deficiency protects bone loss induced by oestrogen deficiency.

Conclusions MiR-146a seems to control bone turnover and miR-146a deficient mice accrue bone over time. Moreover this miRNA has a negative influence on bone loss occurring during oestrogen loss induced osteoporosis. Therefore miR146a could be possibly used as a therapeutic target in the treatment of osteoporosis.

Disclosure of interest None declared

\section{INCREASED GPR22 ACTIVATION TRIGGERS OSTEOARTHRITIS}

${ }^{1}$ L-A Guns*, 'S Monteagudo, ${ }^{2} \mathrm{D}$ Calebiro, ${ }^{3} \mathrm{M}$ Lohse, ${ }^{4} \mathrm{~F}$ Cailotto, ${ }^{1} \mathrm{R}$ Lories. ${ }^{1}$ Skeletal Biology and Engineering Research Centre, KULeuven, Belgium; ${ }^{2}$ Rudolf Virchow Centre, Würzburg; ${ }^{3}$ Rudolf Virchow Centre, University of Würzburg, Germany; ${ }^{4}$ CNRS - Université de Lorraine, UMR7365, Nancy, France

\subsection{6/annrheumdis-2018-EWRR2018.10}

Introduction G protein-coupled receptors (GPCRs) are considered interesting drug targets. GPR22, an orphan receptor, was previously associated to osteoarthritis (OA) in a genome wide association study.

Objectives To investigate GPR22 expression in human healthy and OA cartilage and asses the functional role of GPR22 with in vitro and in vivo models.

Methods GPR22 protein levels were examined by immunohistochemistry. Chondrogenic ATDC5 cells stably overexpressing Gpr22 (GPR22+) and embryonic limb bud cells from mutant Gpr22V385A/V385A mice were cultured as micromasses, and read-outs included gene expression and evaluation of the extracellular matrix. Downstream GPR22 signalling was determined with a PKA activity fluorescence-based assay. Cholecystokinin receptor antagonist AG-041R was tested as GPR22 antagonist. PKA concentrations and kinetics were measured by fluorescence resonance energy transfer (FRET) imaging by PKA sensors in ATDC5 cells. Destabilisation or removal of the medial meniscus (DMM or TMM), for 12 or 8 weeks respectively, was surgically induced in mutant 8 week-old Gpr22V385A/V385A and wild-type C57/B16 mice. Vehicle or AG-041R was injected in the knee joint every 5 days, starting 1 week post-DMM until the mice were sacrificed. Severity was analysed according to OARSI scoring and immunohistochemistry.

Results In healthy human articular cartilage, GPR22 protein was absent, while it is present in OA cartilage. GPR22 + cells showed increased mineralization and decreased proteoglycan content compared to controls after 21 days. Gene expression demonstrated a decrease in Aggrecan and Collagen 2. In contrast, levels of hypertrophic markers Col10 and Mmp13 were increased, suggesting a shift of the cells' differentiation status. PKA activity was reduced in GPR22 + cells compared to controls. The effects on markers, proteoglycan content and mineralization were antagonised by AG-041R. FRET imaging confirmed the neutralising effect of AG-041R on intracellular PKA concentration in GPR22 +cells. In vivo, DMM- and TMM- operated knees of GPR22 V385A/V385A mice showed increased cartilage damage, and increased GPR22 and COL10 expression compared to controls. Intra-articular injections of AG-041R prevented cartilage damage in DMM-induced GPR22 V385A/V385A mice.

Conclusions GPR22 is present in human OA cartilage and not in healthy tissue. Overexpression of GPR22 accelerates chondrocyte hypertrophy. GPR22 can be antagonised by AG-041R. Our preliminary in vivo data show a therapeutic effect of AG$041 \mathrm{R}$ in the DMM model. Thus, GPR22 is genetically and functionally linked to $\mathrm{OA}$ and may be a potential therapeutic target.

Disclosure of interest None declared

\section{WHY JOINT LOCATION MATTERS IN THE PATHOGENESIS OF RHEUMATOID ARTHRITIS}

${ }^{1} \mathrm{M}$ Frank Bertoncelj ${ }^{*},{ }^{1} \mathrm{E}$ Karouzakis, ${ }^{1} \mathrm{~T}$ Masterson, ${ }^{2} \mathrm{C}$ Kolling, ${ }^{1} \mathrm{O}$ Distler, ${ }^{1} \mathrm{C}$ Ospelt. ${ }^{1}$ Center of Experimental Rheumatology, University Hospital Zurich, Schlieren; ${ }^{2}$ Schulthess Clinic, Zurich, Switzerland

\subsection{6/annrheumdis-2018-EWRR2018.11}

Introduction Inflammatory arthritis, such as rheumatoid arthritis (RA) and spondyloarthropaties, follows a characteristic anatomical pattern of joint involvement. We suggest that the local cell types, systemic triggers and site-specific exogenous factors that activate these local cells synergistically contribute to the site-specific occurrence of arthritis. 
Objectives To explore the role of the local stromal cells synovial fibroblasts (SF), in defining the joint-specific synovial biology, relevant for RA.

Methods We studied transcriptomes, epigenomes and functions of hand, shoulder and knee SF from patients with RA or osteoarthritis and from knees of nonarthritic subjects with arthralgia by using RNA-sequencing (Illumina HiSeq 2000, $\mathrm{n}=21$ ), histone ChIP-sequencing (Illumina HiSeq 2500, $\mathrm{n}=7$ ), Infinium HumanMethylation450 BeadChip $(\mathrm{n}=12)$ and in vitro assays for proliferation, adhesion and chemotaxis. We silenced the long noncoding RNA HOTTIP in hand SF using LNA GapmeR, followed by RNA-sequencing $(n=2)$, proteinprotein interaction analysis (STRING) and qPCR confirmation of the HOTTIP target genes. Paraffin embedded RA synovial tissues $(n=48)$ from different joints were scored by the Krenn synovitis score for leukocyte infiltration, synovial lining and density of synovial stroma.

Results The transcriptomes, the global DNA methylation patterns and the histone marks, which mark the actively transcribed DNA regions (H3K27ac) and enhancers (H3K4me1), defined the joint-specific origin of SF. Hand SF showed prominent proliferative, chemotactic and matrix-degrading properties. Hand synovial tissues exhibited increased density of stroma and leukocyte infiltration. The homeobox (HOX) genes that regulate hand embryogenesis (HOXA13, HOTTIP) were the top differentially expressed genes with hand-specific expression in SF. This hand-specific expression pattern coincided with the specific enrichment of the activating histone marks $\mathrm{H} 3 \mathrm{~K} 4 \mathrm{me} 3$ and $\mathrm{H} 3 \mathrm{~K} 27 \mathrm{ac}$ and the absence of repressive H3K27me3 and DNA methylation at the HOTTIP and HOXA13 promoters in hand SF. In contrast, shoulder and knee SF displayed abundant H3K27me3 and DNA methylation, but scarce $\mathrm{H} 3 \mathrm{~K} 4 \mathrm{me} 3$ and $\mathrm{H} 3 \mathrm{~K} 27 \mathrm{ac}$ at the HOTTIP and HOXA13 promoters. Silencing of HOTTIP in hand SF altered the expression of 447 mRNA genes with a $\log$ ratio $>|2|$ $($ FDR $<0.05)$. The HOTTIP regulated genes were strongly enriched in the mitotic cell cycle protein interaction network $\left(n=48\right.$ genes, $\left.p=3.29 \times 10^{-7}\right)$. Several of these genes were confirmed as downregulated by HOTTIP silencing in a larger cohort of hand SF $(n=6, p<0.05)$. Besides, the basal expression of the enriched cell cycle genes, including CDC27 and TADA3, significantly correlated with the basal HOTTIP expression in hand SF $(n=21)$.

Conclusions The IncRNA HOTTIP, which is specifically expressed in hands via epigenetic mechanisms regulates the mitotic cell cycle genes. This might imprint hand SF with an enhanced proliferative potential and might promote the synovial hyperplasia in hand joints, thereby increasing the severity of hand RA.

Acknowledgements IRR-IRF, Promedica, Georg und Berta Schwyzer Winiker Stiftung, CABMM.

Disclosure of interest M. Frank Bertoncelj Grant/research support from: IRR-IRF, Promedica, Georg und Berta Schwyzer Winiker Stiftung, CABMM, E. Karouzakis: None declared, T. Masterson: None declared, C. Kolling: None declared, O. Distler: None declared, C. Ospelt Grant/research support from:
IRR-IRF, Promedica, Georg und Berta Schwyzer Winiker Stiftung, CABMM

\section{JOINT-SPECIFIC SYNOVIAL TGF-B1-INDUCED AXL EXPRESSION DETERMINES THE OUTCOME OF INFLAMMATORY ARTHRITIS BETWEEN ANKLE AND KNEE JOINTS}

CEJ Waterborg*, MGA Broeren, EN Blaney Davidson, MI Koenders, PLEM van Lent, WB van den Berg, PM van der Kraan, FAJ van de Loo. Experimental Rheumatology, Department of Rheumatology, Radboud University Medical Centre, Nijmegen, Netherlands

\subsection{6/annrheumdis-2018-EWRR2018.12}

Introduction Rheumatoid arthritis (RA) manifests in a symmetrical fashion in anatomically distinct synovial joints. The innate immune system, which plays a crucial role in the pathogenesis of RA, is regulated by anti-inflammatory feedback mechanisms such as the tyrosine kinase receptor family TYRO3, AXL and MER. Of importance, AXL is mainly expressed on sentinel cells.

Objectives To investigate the synovial expression of AXL and its putative anti-inflammatory role in arthritis.

Methods KRN serum transfer arthritis was induced in $A x l^{-1-}$ and wild-type mice. Knee and ankle joints were scored macroand microscopically. Synovial gene and protein expression of $A x l$ was determined in naive and TGF- $\beta 1$-overexpressing joints. AXL expression was determined in M1-like or M2-like macrophages and RA synovium. Human macrophages and synovial micromasses were stimulated with TGF- $\beta 1$ or the AXL inhibitor R428.

Results The ankle joints of $A x l^{-/-}$mice showed an increased macroscopic disease score during arthritis development $(p<0.001$ at day 7). In agreement with this, ankle joints of $A x l^{-/-}$mice showed exacerbated arthritis pathology whereas no effect of $A x l$ gene deletion was observed on gonarthritis pathology. To explain this spatial difference, we examined the synovium of naive mice. In contrast to the knee, the ankle synovial cells prominently expressed AXL. Moreover, the M2like macrophage phenotype was the dominant cell type in the naive ankle joint. Human M2-like macrophages expressed higher levels of AXL and blocking AXL increased their inflammatory response. In the murine ankle synovium, gene expression of $T g f b 1$ was increased and Tgb1 correlated with $A x l$ (Pearson $\mathrm{r}=0.8860, \mathrm{p}=0.0012)$. Moreover, TGFB1 and AXL expression also correlated in human RA synovium (Pearson $\mathrm{r}=0.7233, \mathrm{p}=0.0035$ ). In human macrophages and synovial micromasses, TGF- $\beta 1$ enhanced AXL expression. Moreover, TGF- $\beta 1$ overexpression in naive murine knee joints induced synovial AXL expression.

Conclusions Differences in synovial AXL expression are in accordance with the observation that AXL dampens arthritis in ankle, but not in knee joints. We provide evidence that the local differences in AXL expression could be due to TGF- $\beta 1$, and suggest similar pathways operate in RA synovium.

Disclosure of interest None declared 